\title{
LA PROLIFERACIÓN DE ARMAS DE DESTRUCCIÓN MASIVA EN LA ESTRATEGIA DE SEGURIDAD NACIONAL 2013
}

\author{
Alberto Priego ${ }^{1}$ \\ Universidad Pontificia de Comillas / UNISCI
}

\begin{abstract}
Resumen:
Las Armas de Destrucción Masiva son una de las mayores amenazas que tendrá que afrontar la humanidad en las próximas décadas. Si el siglo XX estuvo marcado por el poder de las armas nucleares el siglo XXI será el siglo de las armas químicas y bacteriológicas. A pesar de los regímenes internacionales que se han establecido para el control de la proliferación de las Armas de Destrucción Masiva la realidad es que éstos no han funcionado. Esta inquietante realidad ha sido abordada con dificultades por la Estrategia Española de Seguridad Nacional que en mi opinión no le ha concedido la importancia que merece.
\end{abstract}

Palabras clave: Proliferación, Estrategia de Seguridad Nacional, Armas de Destrucción Masiva, TNP.

Title in English: "Mass-Destruction Weapons Proliferation in the National Security Strategy 2013"

\section{Abstract:}

Weapons of Mass Destruction are of the most important threats the humankind has to face in the next decades. If the 20th Century was marked by the power of nuclear weapons, the 21st century will be the century of chemical and bacteriological ones. Despite the international regimes established to manage the proliferation of weapons of mass destruction, the reality is that these initiatives have not worked. In my opinion, this disturbing reality has dealt with difficulties by the Spanish National Security Strategy The Spanish Government should have realised the importance that this challenge deserves.

Keywords: Proliferation, National Security Strategy, Weapons of Mass Destruction, NPT.

Copyright @ UNISCI, 2014.

Las opiniones expresadas en estos artículos son propias de sus autores, y no reflejan necesariamente la opinión de UNISCI. The views expressed in these articles are those of the authors, and do not necessarily reflect the views of UNISCI.

\footnotetext{
${ }^{1}$ El Dr. Alberto Priego es profesor propio adjunto en el Área de Ciencia Políticas (Departamento de Disciplinas Comunes) en la Universidad Pontificia Comillas (ICADE), ha sido investigador invitado en la School of Oriental and African Studies (The University of London), es investigador senior de UNISCI y es miembro del Foro Hispano-Argelino.

E-Mail: apriego@upcomilllas.es. 


\section{Introducción}

El tema de la proliferación de Armas de Destrucción Masiva (ADM) es un elemento especialmente sensible para la Sociedad Internacional. En los últimos años se ha incrementado su disponibilidad. Muchos Estados, 24 según algunos autores², están en disposición de poder desarrollar este tipo de armas. El poder de destrucción de las Armas de Destrucción Masiva (ADM) es tal que las convierten en una herramienta que debe ser cuidada con especial delicadeza ${ }^{3}$. Una de las frases que mejor recoge su capacidad de destrucción y por lo tanto el temor que despiertan sea la pronunciada por Madeleine Albright "Each night we knew that within minutes, perhaps through a misunderstanding, our world could end and morning never come ${ }^{4}$.

Por su poder de destrucción y el temor que generan, algunos autores, como Terry Terrif, han señalado cuatro grandes problemas de Armas de Destrucción Masiva (ADM):

a) Su alto coste: Las Armas de Destrucción Masiva (ADM) son caras de adquirir, mantener e incluso de destruir.

b) Son peligrosas: gracias al inmenso poder de destrucción de las Armas de Destrucción Masiva (ADM), especialmente de las nucleares, hacen que estos dispositivos puedan contribuir a alterar el equilibrio de poder los Estados generando así incertidumbre y desconcierto.

c) No aceptadas por todos los Estados: Aquellos Estados que no poseen armas nucleares se pueden sentir inferiores y desvalidos frente a las potencias nucleares. En algunos casos como, India, Pakistán o Israel, los Estados se pueden ver tentados a desarrollar Armas de Destrucción Masiva (ADM) para asegurarse su supervivencia.

d) Intrínsecamente inestables: El último de los problemas de las Armas de Destrucción Masiva (ADM) es la inestabilidad. De puertas para fuera ya hemos mencionado que genera incertidumbre pero en el plano interior hay que ser especialmente cuidadosos ya que un colapso del sistema político de las potencias nucleares podría derivar en un conflicto nuclear de dimensiones mundiales.

\section{Definición del problema}

La primera vez que encontramos el término Armas de Destrucción Masiva (ADM) fue en 1937. El entonces Arzobispo de Canterbury Cosmos G. Lang que apelaba a la Responsabilidad Cristiana para acabar con el horror ${ }^{5}$ generado por los bombardeos en España

\footnotetext{
2 "The nine current members of the nuclear club still possess about 27000 operational nuclear weapons between them. At least 15 countries, have on hand enough highly enriched uranium for a nuclear weapon", en Siracusa, Joseph M. (2008): Nuclear weapons. A Very Short introduction, Oxford, Oxford University Press, p. II.

3 "Nuclear deterrence was a form of security for individual states in which every state and society was vulnerable to being completed destroyed", en Terrif, Terry; Croft, Stuart; James, Lucy and Morgan, Patrick M. (2007): Security Studies Today, Cambridge, Polity Press, p. 62.

${ }^{4}$ Coleman, David and Siracusa, Joseph M. (2006): Real-world Nuclear Deterrence: The Making of International Strategy, Westport, Praeger, p. 6.

5 Carus, Seth W.: "Defining "Weapons of Mass Destruction", Center for the Study of Weapons of Mass Destruction (National Defence University), Ocassional Paper, $\mathrm{n}^{\circ}$ 8, p. 7, en http://www.ndu.edu/press/lib/pdf/CSWMD-OccasionalPapers/CSWMD_OccationalPaper-8.pdf.
} 
y en China por parte de la aviación Alemana y Japonesa respectivamente. Si bien en dichos acontecimientos no se utilizaron agentes químicos, bacteriológicos o nucleares, su capacidad de destrucción mereció tal calificativo.

El término ADM con las implicaciones que hoy lo conocemos se lo debemos según James Goodby (Brookings Institution) a Vanevar Bush quien en su condición de asesor presidencial elaboró la expresión "weapons adaptable to mass destruction" le dio el significado actual. No obstante es cierto que Cosmos G. Lang ya hizo alguna alusión a las ADM en relación a las armas químicas utilizadas por los italianos en la Guerra de Abisinia.

En 1948 la Comisión de las Naciones Unidas sobre Desarme Convencional instó al Consejo de Seguridad a desarrollar una definición de AMD. El Consejo de Seguridad estableció que por que ADM se estendía "weapons of mass destruction should be defined to include atomic explosive weapons, radio active material weapons, lethal chemical and biological weapons, and any weapons developed in the future which have characteristics comparable in destructive effect to those of the atomic bomb or other weapons mentioned $a^{a b o v e}{ }^{6}$ " La definición fue vetada por la URSS hasta los años 70 lo que permitió que la Asamblea General aprobara una resolución donde finalmente se consensuaba la definición ${ }^{7}$.

Los Estados Unidos han desarrollado una interesante evolución en la definición de las ADM. Recientemente, los Estados Unidos a través de su Departamento de Defensa ha redefinido el concepto para adaptarlo a las circunstancias. Este debate comenzó en 2005 cuando el Departamento de Defensa a través de su DOD Dictionary of Military and Associated Terms definió las Armas de Destrucción Masiva como "capable of a high order of destruction and/or of being used in such a manner as to destroy large numbers of people ${ }^{8 \text { " }}$ Sin embargo, si bien es cierto que al decir high order of destruction se incluyen artilugios usados en el campo de batalla como morteros o granadas posteriormente se aclara la naturaleza de estas armas "can be high explosives or nuclear, biological, chemical, and radiological weapons"

También la URSS y posteriormente Rusia han desarrollado una importante doctrina en lo que a la definición de las ADM se refiere. Quizás la primera referencia la encontremos en el término Oruzhiye massovogo porazheniya que tenía la implicación de armas que podían infligir un alto daño incluyendo agentes nucleares, químico, radilógicos y bacteriológico. Esta acotación se mantiene en las doctrinas militares de 1993, 2000 y 2010 con algunas pequeñas especificidades. En la Doctrina militar de 1993 menciona las armas nucleares, biológicas y bacteriológicas así como "nuevos tipos" de ADM que puedan aparecer. En las dos doctrinas posteriores sin desaparecer las alusiones a las químicas y las bacteriológicas sí que es cierto que se centran en las nucleares, despareciendo en todo caso la alusión a esos nuevos tipos.

En todo caso podemos establecer que por Armas de Destrucción Masiva debemos entender aquellas que poseen gran capacidad de destrucción y que conlleva la utilización de elementos nucleares, químicos ${ }^{9}$ o bacterológicos. Hay otros conceptos que también se han

\footnotetext{
${ }^{6}$ Ibid., p. 10.

${ }^{7}$ Resolución Asamblea General, /36/84.

${ }^{8}$ Ibid., p. 2.

${ }^{9}$ En la última década se ha incrementado de forma exponencial la industria química, especialmente en Asia. Sobre las perspectivas de futuro ver informe: "Chemical Industry vision 2030. European Perspective", At Kearney, en http://www.atkearney.com/documents/10192/536196/Chemical+Industry+Vision+2030+A+European+Perspecti ve.pdf/7178b150-22d9-4b50-9125-1f1b3a9361ef.
} 
utilizado como CBR (Chemical, Biological and Radiological) NBC (Nuclear Biological and Chemical) o $\mathrm{CBRN}^{10}$ (Chemical, Biological, Radiological and Nuclear) No obstante quizás sean sus siglas en inglés WMD o ADM en castellano las que se han impuesto finalmente ${ }^{11}$ que además nos permiten trabajar con un concepto consensuado.

Centrándonos en los documentos que estamos analizando, la Estrategia Española de Seguridad Nacional, lo primero que nos encontramos nada más comenzar el apartado relativo a la Proliferación de Armas de Destrucción Masiva (ADM) es una buena definición del concepto. Lo único que encontramos es una de definición ambigua y poco concisa de este tipo de dispositivos. Vemos que se señala tanto a unos tipos de armas determinados (nucleares, químicas y bactereológicas) como a los dispositivos de lanzamiento (misiles balísticos como de crucero). Este párrafo nos permite hacer algunos comentarios previos.

A. Las armas de destrucción masiva se circunscribe a tres tipos. Las las nucleares, las químicas y las bactereológicas. Vemos aquí el primer cambio con la estrategia aprobada en 2011. En esta última también se incluían las radiológicas. Sobre este punto hay que decir que la terminología no es clara. Podemos encontrar muchas denomnaciones que irían desde arma radiológica, bomba radiológica o bomba sucia. Quizás el más correcto sea Dispositivo de Dispersión Radiológica. Un artefacto de carácter convencional que es detonado junto a una fuente radiactiva. Este tipo de artefactos no suele ser incluido entre las armas de destrucción masiva y su inclusión en el documento de 2011 implica la inclusión de las acciones de grupos terroristas ya que son los verdaderos usuarios de este tipo de $\operatorname{armas}^{12}$.

B. A continuación señala a los sistemas de lanzamiento haciendo una alusión a los misiles balísticos y de crucero. Respecto de los misiles balísticos no señala su alcance por lo que nos suscita dos dudas:

1. Por un lado se refiere a misiles balísticos intercontinentales estaríamos hablando de misiles con capacidad de más de $5.500 \mathrm{~km}$. En este sentido estaríamos señalando a dispositivos de lanzamiento ICBM, SLBM o bombarderos estratégicos que estarían en posesión de Aliados de la OTAN (EEUU, Reino Unido y Francia) China o Rusia.

2. Por otro lado nada se dice de los misiles de alcance intermedio que son los verdaderos problemas a nivel internacional por ser los que poseen aquellos Estados que pueden desestabilizar el mundo (Pakistán, Corea del Norte o India).

Llama la atención la ausencia en el documento de 2011 y en el de 2013 de alusiones a los dispositivos minutiarizado, un problema cada vez más común que hace del mundo un lugar bastante más inseguro. Precisamente esa fue la intención que tenía la revisión del concepto llevada a cabo por los Estados Unidos y que dio lugar a esa nueva definición del Dictionary of

\footnotetext{
${ }^{10}$ La OTAN en su documento: “NATO's Comprehensive, Strategic-Level Policy for Preventing the Proliferation of WMD and Defending against, Chemical, Radiological and Nuclear (CBRN) Threats".

${ }^{11}$ Sobre una historia de los términos utilizados para las Armas de Destrucción Masiva (ADM), ver: Shafire, William: "On Language; Weapons Of Mass Destruction”, The New York Times Magazine. 19 de abril de 1998, en http://www.nytimes.com/1998/04/19/magazine/on-language-weapons-of-mass-destruction.html.

${ }^{12}$ El Terrorismo Checheno usó un DDR en 1995 en el atentado de Izmailovsky usando Cesio 137.
} 
Military and Associated Terms llevada a cabo por el Departamento de Defensa que hemos mencionado anteriormente.

Otro de los elementos conceptuales que merecen ser destacados es la ausencia de un glosario con los términos más relevantes. Por ello, sería conveniente la introducción de un apéndice sobre términos relacionados con la proliferación nuclear en la línea desarrollada por los Estados Unidos en la Joint Publication on Combating Weapons of Mass Destruction. Este documento no sólo es rico en terminología sino que aporta nuevas dimensiones a la proliferación que permiten conocer en profundidad este fenómeno. Cabe destacar diferencias terminológicas tales como "counter-proliferation" frente a "non-proliferation" o "weapons of mass destruction elimination" frente a "weapons of mass destruction interdiction"13. La inclusión de este apéndice aportaría mayor precisión al documento e iría en la línea de otros llevados a cabo por Estados aliados.

Otro de los aspectos que más llaman la atención en estos primeros párrafos del documento es la asunción de la existencia de una Comunidad Internacional. Resulta cuanto menos paradógico que hablando de Armas de Destrucción Masiva se pueda utilizar dicho término. Por ello quizás hubiera sido más interesante otros como Sociedad Internacional o incluso Sistema Internacional. Siguiendo a Barry Buzan la Sociedad es una respuesta a la existencia de un sistema e implicaría el reconocimiento de una interdependencia así como la existencia de unas reglas que evitarían los $\operatorname{conflictos}^{14}$. En definitiva hablar de Comunidad Internacional parece especialmente cuanto menos utópico, sobre todo cuando estamos tratando de Armas de Destrucción Masiva que son en sí mismas una ausencia de valores entre los Estados.

\section{Implicaciones Internacionales}

A nivel internacional podríamos hablar de las acciones llevadas a cabo dentro del régimen de no proliferación así como de las políticas de las organizaciones de seguridad en las que España está integrada.

En primer lugar vamos a analizar la alusión directa que se hace a "los regímenes de los tratados internacionales" 15 . Si bien es cierto que no se señalan directamente al régimen de proliferación nuclear, química y bacteriológica parece claro que se refiere a estos regímenes.

\footnotetext{
13 Joint Chiefs of Commanders (2009): Joint Publication on Combating Weapons of Mass Destruction. Joint Chief of Commanders, Washington D.C, p. 5, en http://www.fas.org/irp/doddir/dod/jp3 40.pdf.

${ }^{14}$ Buzan, Barry: "New Patterns of global security in the twenty-fist century", International Affairs, vol. 67, no 3 (1991), p. 438.

${ }^{15}$ Sobre el Régimen de No-Proliferación Nuclear ver: Bolton, John: "Is There Really 'Law' in International Affairs", Transnational Law and Contemporary Problem, vol. 10, nº 1 (2000); Burroughs, John: "The Man Who Averted Nuclear War", DisarmamentActivist.org (19 de enero del 2006); Garrido, Vicente: "El futuro del desarme y la no proliferación”, Política Exterior, vol. 19, nº 105 (2005); Garrido, Vicente: “Acuerdo de Revisión del TNP. La guerra de las galaxias (II): ¿La amenaza fantasma?”, El País, 22 de mayo de 2000, en http://elpais.com/diario/2000/05/22/internacional/958946423 850215.html; Jones, Rodney W. y Mark G. McDonough (1998): Tracking Nuclear Proliferation: A Guide in Maps and Charts, Washington, Carnegie Endowment for International Peace; Sanger, David E: "Month of Talks Fails to Bolster Nuclear Treaty", New York Times, 28 de mayo del 2005, en

http://www.nytimes.com/2005/05/28/politics/28nations.html?pagewanted=print.
} 
En lo que a las organizaciones internacionales se refiere vamos a analizar las menciones a la Unión Europea y a la OTAN esencialmente. La inclusión de la UE en el documento de 2013 debe ser vista como muy positiva ya que en el documento de 2011 no se mencionaba. Sin embargo, llama la atención la ausencia de mención a la Estrategia Europea de Seguridad y a la "Declaración sobre la No-Proliferación de Armas de Destrucción Masiva" aprobada en Consejo Europeo de Tesalónica de $2003^{16}$. A pesar de estas graves carencias, el documento sí que menciona "la Estrategia contra la Proliferación de Armas de Destrucción Masiva de la UE" y las Conclusiones del Consejo de 2008 donde se aprueban "Las Nuevas líneas de actuación de la UE en la lucha contra la proliferación" de armas de destrucción masiva y sus vectores". Este documento está dedicado en su totalidad a marcar nuevas líneas de actuación en la lucha contra la proliferación y sistemas de transporte ${ }^{17}$. Probablemente este documento debería haberse mencionado en la sección de la estrategia donde se explican las líneas de actuación. Junto con esta carencia también destaca la ausencia de al Plan de Acción para la Implementación de los principios básicos.

Para concluir con las alusiones a la UE, parece echarse en falta alguna referencia a los esfuerzos negociadores de la UE con Irán, a quien por otra parte atribuye sin duda el desarrollo de un programa nuclear de carácter militar.

Para la Estrategia de Seguridad Nacional la OTAN, es una organización internacional que está prácticamente ausente en lo que a la no-proliferación se refiere. La Alianza Atlántica ha desarrollado un trabajo muy efectivo en el campo de la no-proliferación ${ }^{18}$, por lo que llama la atención la casi nula mención a la Alianza en la Estrategia de Seguridad Nacional. Por encima del resto, lo más sorprendente es la omisión a los Conceptos Estratégicos de Roma (1992), Washington (1999) y Lisboa (2010) ya que en todos ellos se hacen menciones expresas a la proliferación nuclear. También es llamativo que dentro de las denominadas líneas de actuación ni siquiera se mencione el "NATO Multinational Chemical, Biological, Radiological and Nuclear Defence Battalion" creado en 2002 en la Cumbre de Praga y del que España forma parte.

También llama la atención la ausencia de mención de otro documento vital para la no proliferación: "NATO's Comprenhensive, Strategic-Level Policy for Preventing the Proliferation of WMD and Defending against CBRN Threats ${ }^{19}$ " Este documento ha servido como guía de las acciones de la OTAN en este campo y debería orientar las acciones de España en este campo. Tampoco hay mención a la Proliferation Security Initiative, un programa en la que España ha tenido un rol importante. En cambio sí que se mencionan otros puntos como el Escudo Antimisiles de la OTAN en el que España a través de las instalaciones de Rota tiene gran protagonismo.

Otro de los aspectos que más llaman la atención es la ausencia absoluta de referencias a los esfuerzos llevados a cabo por las Naciones Unidas en el marco de la lucha contra la proliferación de Armas de Destrucción Masiva (ADM) Solamente encontramos una referencia

\footnotetext{
${ }^{16}$ Council of the European Union: "Presidency conclusions", Thessaloniki, (19 у 20 de junio del 2003),en http://europa.eu/rapid/press-release_DOC-03-3_en.htm.

${ }^{17}$ Council of the European Union: "Council Conclusions and new lines for actions by the European Union in combating the proliferation of weapons of mass destruction and their delivery systems" 17172/08, en http://register.consilium.europa.eu/doc/srv?l=EN\&t=PDF\&gc=true\&sc=false \&f $=$ ST $\% 2017172 \% 202008 \% 20 \mathrm{INI}$ T\&r=http\%3A\%2F\%2Fregister.consilium.europa.eu\%2Fpd\%2Fen\%2F08\%2Fst17\%2Fst17172.en08.pdf.

18 Terzuolo, Eric, C: "Combating WMD proliferation", NATO Review (Otoño del 2005), en http://www.otan.nato.int/docu/review/2005/issue3/english/art3.html.

${ }^{19}$ Disponible en http://www.nato.int/cps/en/natolive/official texts 57218.htm?selectedLocale=en.
} 
en las "Líneas de Acción Estratégicas" a la Resolución 1540 de 2004. Sin embargo no se menciona ni el comité 1540 ni otras resoluciones como la 1673 (2006), la 1810 (2008) o la 1977 (2010).

Las alusiones a las armas químicas y bactereológicas son escasas y se reducen a un simple párrafo. Se podría decir que hay una clara asimetría respeto del espacio dedicado a las armas nucleares que aglutina más espacio en el documento de 2013. En una línea similar se podría seguir el ejemplo de otros documentos desarrollados por nuestros aliados donde se hace especial hincapié en este tipo de armas. Me estoy refiriendo a documentos como "National Strategy for Countering Biological Threats" ${ }^{20 "}$ o la "Chemichal Biological Strategic Plan $^{2 l "}$ " desarrolladas por la Casa Blanca y por el Departamento de Defensa de los Estados Unidos.

En esta misma línea quizás hubiera sido necesaria algunas alusiones a los esfuerzos llevados a cabo por los Estados Unidos en materia de desarme. Desde el famoso discurso del Presidente Obama en Praga hasta la firma del nuevo START ${ }^{22}$ pasando por la Revisión del TNP $^{23}$, Washington está llevando a cabo importantes esfuerzos para acabar con la proliferación nuclear. Sin embargo, no podemos olvidar que Rusia sigue confiando su fuerza en el mantenimiento de las armas nucleares frente a una OTAN que se expande cada vez más y con un ejército que necesita fuertes inversiones para volver a ser un ejército moderno. El problema es que la viabilidad del TNP está llegando a ser cuestionable por el incumplimiento de las condiciones establecidas para su extensión definitiva en 1995, el proceso de modernización de los arsenales nucleares, frente a lo que establece el artículo 6, la existencia de Estados nucleares que no han firmado el NPT, como Pakistán, la India, Israel, o que se han retirado del tratado, como la República Popular Democrática de Corea, creando todos ellos incentivos muy importantes para la proliferación de sus vecinos, caso de Pakistán sobre Irán, de la República Popular Democrática de Corea sobre los Estados no nucleares del nordeste de Asia o de Israel con sus vecinos árabes.

Hay una alusión a los grupos no estatales, esencialmente terroristas ${ }^{24}$, y la posibilidad de que se pudieran hacer con armas de destrucción masiva. En el documento de 2011 se señala directamente a los grupos yihadistas y a AlQaeda como posibles poseedores de este tipo de armas. En el de 2013 no se hace ninguna mención expresa. Otra diferencia es que en 2011 se habla de dos zonas de riesgo para la proliferación, Asia Central y el Sahel, en el de 2013 no se señala ninguna. Quizás se echa en falta alguna mención a Pakistán sobre todo teniendo en

\footnotetext{
${ }^{20}$ National Security Council (2009): National Strategy for Countering Biological Threats” National Security Council, Washington D.C.

${ }^{21}$ Department of Defence (2008): Chemical Biological Research Program, Department of Defence, Washington D.C.

${ }^{22}$ La cooperación con Rusia en material de lucha contra la proliferación es fluida. Prueba de esta buena relación es la cooperación dentro del Consejo OTAN Rusia llevada a cabo desde el NRC (Working Group on Arms Control, Disarmament and Non-Proliferation - NRC(ADN) 00000000).

${ }^{23}$ Podemos ver que las últimas revisiones del TNP han sido diferentes. Mientras que en 1995 y 2000 se producen algunos avances, en el año 2005 la confrontación es muy grande. La retirada del ABM de los Estados Unidos complica las negociaciones. En 2010 se habla de la firma del nuevo START y del discurso de Obama en Praga. Frente a esto encontramos otras posturas como la modernización del arsenal nuclear chino y el temor ruso a un escudo antimisiles.

${ }^{24}$ Garrido, Vicente: “Terrorismo nuclear: ¿desafío a la seguridad?”, Política Exterior, vol. 26, no 148 (2012).
} 
cuenta que las acciones llevadas a cabo por el Doctor A.Q. Khan ${ }^{25}$ son consideradas especialmente preocupantes para otros Estados como Estados Unidos ${ }^{26}$ o Reino Unido ${ }^{27}$.

La obtención de armas de destrucción masiva por terroristas es catalogada como riesgo y amenaza al mismo tiempo. Esta unión fue calificada por Richard Lugar como "vertex of evil" 28 . En el siguiente apartado analizaremos esta diferenciación con mayor detalle, sin embargo, sería conveniente resaltar que los actores no estatales que están potencialmente implicados en el ciclo de la proliferación no pueden ser reducidos a los grupos terroristas. Se debe tener en cuenta, por supuesto a grupos terroristas, pero también a organizaciones de crimen internacional, Rogue-Scientist/Technicians, extremistas e incluso individuos que actúan por en solitario ${ }^{29}$.

Por último, se puede decir que el documento concede una excesiva importancia a los actores no estatales. Si bien es cierto que cada vez más este tipo de actores están más cerca de la obtención de armas de destrucción masiva, hoy por hoy, la principal amenaza siguen siendo estatal. Estados como Corea del Norte $^{30}$ y en el pasado Libia, Siria e Irán se configuraban con una principales sospechosos de proliferar. Por el contrario, se debería hacer una incidencia mayor en la cooperación ilícita de algunos estados con actores no estatales para la consecución de armas de destrucción masiva. En esta misma línea, no debería ser tratado con tanta ligereza la importancia de los Estados Fallidos en la proliferación de armas de destrucción masiva ya que en otros documentos similares, los Estados Fallidos ocupan un rol muy importante en las preocupaciones de seguridad.

\section{Calificación del riesgo/amenaza}

Tanto en la Estrategia Española de Seguridad de 2011 como en la Estrategia de Seguridad de Nacional de 2013 hay una clara confusión o cuando menos indeterminación entre los conceptos riesgos y amenazas. Si bien es cierto que este es un punto muy debatido por la doctrina de estudios de seguridad, no parece acertado utilizar los dos conceptos como si fueran sinónimos. Como hemos dicho anteriormente este error se comete en el documento de 2011 y en menor medida en el 2013.

Uno de los elementos que quizás se echan en falta en el documento que estamos analizando sea la división entre "Tradicional WMD" y "Non-Traditional WMD". Podemos

\footnotetext{
${ }^{25}$ Sobre las acciones llevadas a cabo por A.Q Khan, ver Garrido, Vicente: "Pakistán y el doctor Khan", Política Exterior, vol. 18, nº 98 (2004).

${ }^{26}$ Una mención especial a la red del científico pakistaní A.Q Khan se puede encontrar en: Joint Chiefs of Commanders (2009): Joint Publication on Combating Weapons of Mass Destruction, Washington D.C, p. 40, en http://www.fas.org/irp/doddir/dod/jp3 40.pdf.

27 "Pakistan is a country of particular concern, having spawned the nuclear proliferation network run by A.Q. Khan which helped to supply the illicit nuclear weapons programmes of Iran, Libya, North Korea and possibly other states", en "Global Security: Non-Proliferation", House of Commons Foreign Affairs, Parliament of the UK-Fourth Report of Session 2008-09, p. 103.

28 "we are facing a -vertex of evil- an intersection of WMD and Terrorism" Lugar, Richard G: "Redefining NATO's mission: Preventing Weapon of Mass Destruction”, Washington Quaterly, vol. 25, n 3 (Summer 2002), p. 10.

${ }^{29}$ Joint Chief of Staff (2006): "National Military Strategy to Combat Weapons of Mass Destruction" Washington D.C, JCS (13 February 2006), p. 9, en http://www.defense.gov/pdf/nms-cwmd2006.pdf.

30 Sobre las actividades de Corea del Norte ver: Abad, Gracia y Priego, Alberto: "Inteligencia y Contraproliferación. Las Relaciones entre Corea del Norte y Pakistán”, Inteligencia y Seguridad. Revista de Análisis y Prospectiva, no 4 (Julio-noviembre 2008).
} 
hablar de dos elementos fundamentales que marcan la diferencia entre las Armas de Destrucción Masiva convencional y la no-convencional.

A. Los Medios de transporte/uso: Mientras que las armas tradicionales de destrucción masiva utilizan medios convencionales tales como misiles, cohetes, aviones etc...las armas de destrucción masiva no convencionales hacen alusión a dispositivos no militares tales como mochilas, sprays o correo postal que se transportan por medios privados (coches, motocicletas) o públicos (tren, metro, avión o autobús).

Cada vez es más frecuente el uso de nano tecnologías para la construcción, transporte o ensamblaje de elementos necesarios para la proliferación de Armas de Destrucción Masiva. Una mención especial merecen los dispositivos aéreos no tripulados, que en los próximos años se convertirán en habituales. Por esta razón los UAS deberán ser objeto de especial atención en la lucha contra la proliferación de armas de destrucción masiva ya que su detección resulta complicada y sus efectos pueden ser devastadores.

B. Los objetivos: en la Proliferación Tradicional de las Armas de Destrucción Masiva, en principio los objetivos son militares o al menos relacionados con los poderes públicos. Sin embargo, en el caso de la proliferación no convencional se amplían los objetivos incluyendo centros comerciales, congregaciones religiosas o colegios y hospitales.

Esta diferenciación que resulta crucial para combatir este problema es obviada en los documentos que guiarán la acción de los poderes públicos en España en materia de seguridad.

\section{Líneas de Actuación}

El objetivo de las líneas de actuación que destaca en la Estrategia de Seguridad Nacional no queda del todo claro. Por un lado se habla de impedir la proliferación y por el otro de evitar el acceso a sustancias peligrosas por parte de terroristas y criminales. Por un lado, hay gran confusión respecto de si el objetivo es solamente evitar que grupos no estatales accedan a "sustancias" o precursores para la fabricación de armas de destrucción masiva, esencialmente químico, biológico o nuclear, o si por el contrario también estamos trabajando frente a Estados. Es decir no se aclara si el objetivo también se extiende a Estados que puedan proliferar en un momento dado.

La primera línea de actuación es muy extensa y se menciona el multilateralismo en el seno de la OTAN y de la UE. De nuevo se habla de terrorismo y actores no estatales descartando por tanto a los Estados. Se habla también de "medidas preventivas de vigilancia" que resulta a todas luces confusa ya que no se entiende si se está refiriendo a medidas de alerta temprana o de inteligencia.

La segunda línea de actuación es el fortalecimiento del sistema (régimen) de noproliferación lo cual es coherente con el resto de la estrategia y con los fines de la UE y de la OTAN. El Proyecto de Estrategia Europea de Seguridad Global también hace mención a la proliferación y a su conexión con otros problemas de seguridad como el terrorismo o el crimen organizado ${ }^{31}$.

\footnotetext{
31 "By working with both state and non-state partners in the region, the EU should therefore upgrade its efforts to combat international terrorism, the proliferation of weapons of mass destruction, organized crime and the
} 
La tercera línea se refiere al ámbito interno con el fin de evitar la proliferación en el interior. Por ello, se habla incluso de un plan de biocustodia. La amenaza interna no ha sido mencionada anteriormente lo que implica una cierta falta de coherencia. Esta línea de acción estaría dentro de las marcadas por el Plan de Acción de la UE y de la PSI ${ }^{32}$.

El resto de las medidas están destinadas a la transferencia de material fisible y potencial para desarrollar armas de destrucción masiva. Resulta curioso que se señalen estos puntos cuando en la estrategia no se habla de los compromisos internacionales adquiridos por España en materia de transferencias nucleares, químicas y bacteriológicas.

Destacamos la sexta línea de actuación donde se menciona expresamente algunas iniciativas en la que España está colaborando. Sin embargo, si bien es cierto que resulta de gran interés este tipo de cooperación, cabe preguntarse porque no se menciona de forma explícita otras formas de cooperación institucionalizadas en las que también colabora España. En concreto me estoy refiriendo a la PSI o las acciones llevadas a cabo en el marco de OTAN o de la UE. Por ello, en el plano de las líneas de acción estratégicas podemos afirmar que si bien tocan aspectos muy importantes de la no-proliferación no recoge los foros y los instrumentos más efectivos de este campo.

\section{Comparación con otras Doctrinas de Seguridad Nacional}

Esencialmente podemos comparar la estrategia española con los documentos aprobados por la República Francesa, por los Estados Unidos y por el Reino Unido o por Australia. Mientras que el primero acaba de aprobar un documento, el segundo está en vías de hacerlo y el Reino Unido se rige por uno de 2010. Veamos como tratan el asunto de la proliferación de armas de destrucción masiva en estos documentos que hemos mencionado anteriormente.

\subsection{Livre Blanc. Défénse et Sécurité Nationale 2013}

El documento de seguridad francés es muy claro con la amenaza ${ }^{33}$ que plantean las armas de destrucción masiva y su vinculación al terrorismo ${ }^{34}$. Como solución a este problema plantea como única posibilidad la cooperación multilateral en el marco europeo manejando una cuidada ambigüedad sobre las relaciones con los Estados Unidos ${ }^{35}$.

En este sentido Francia expresa su voluntad de lograr en la medida de lo posible y siempre desde la responsabilidad un compromiso de desarme siguiendo el artículo 6 del Tratado de No Proliferación Nuclear (TNP) ${ }^{36}$ En esta misma línea aboga por la prohibición de

trafficking of arms, drugs and human beings" VV.AA. (2013): EU Global Strategy towards a European Global Strategy, p. 12, disponible en www.europeanglobalstrategy.eu.

32 "The WMD strategy called on member states to adopt common policies related to the criminal punishment for exporting, brokering and smuggling WMD-related material", en Rhode, Ben: "WMD Proliferation" en Giegerich, Bastian (2011): Europe And Global Security, London, IISS-Routledge, p.156.

${ }^{33}$ Distingue de forma adecuada entre riesgo y amenaza.

34 "Livre Blanc de la Défense et la Sécurité: La protection contre le risque nucléaire, radiologique, biologique, chimique ou explosif (NRBC-E)", Ministerio de defensa, París, (2013), p. 106.

35 "En matière de lutte contre la prolifération d'armes de destruction massive, la France participe activement à l'action multilatérale, notamment à celle conduit au niveau européen pour harmoniser les dispositions visant à criminaliser les activités proliférantes et accrô̂tre ainsi l'effet dissuasif commun”, en Ibid., p. 106.

36 "À ce titre, elle oeuvre activement en faveur d'un "désarmement général et complet sous un contrôle strict et efficace », objectif fixé par l'article VI du Traité sur la non-prolifération des armes nucléaires (TNP)”, en Ibid., p. 75 . 
las pruebas nucleares así como de la transferencia de materia fisible, una vez Francia concluyó su programa de pruebas nucleares en el Pacífico Sur ${ }^{37}$.

\subsection{National Security Strategy 2011}

En el documento aprobado por la Casa Blanca se señala que no existe una amenaza mayor que la proliferación nuclear sobre todo cuando ésta procede de un actor no estatal ${ }^{38}$. La preocupación está motivada por un lado por los grandes stocks de armas nucleares desarrolladas durante la Guerra Fría y por otro lado por la emergencia de grupos no estatales vinculados con el terrorismo o con el crimen transnacional con pretensiones nucleares ${ }^{39}$.

Estados Unidos reconoce la magnitud del problema y asume que no puede solucionarse sin la cooperación multilateral. Por ello, al menos en la Estrategia de Securidad Nacional los Estados Unidos plantean el fortalecimiento del régimen de no proliferación nuclear ${ }^{40}$.

Por otro lado, se refuerza el deseo del Presidente Obama de alcanzar a medio plazo el Cero Nuclear, algo que ya señaló en su famoso discurso de Praga ${ }^{41}$ en 2009. Incluso se señala la conveniencia de la plean ratificación del CTBT e incluso se va más allá buscando la firma de un nuevo tratado que regule las transferencias de materia fisible ${ }^{42}$.

En todo caso los Estados Unidos, de acuerdo al propio Tratado de No Proliferación Nuclear, reconoce el derecho de los Estados a usar la energía nuclear ya que es más limpia, barata o ecológica ${ }^{43}$.

Por último, se señala el riesgo que plantean las armas biológicas para la población y por ello se sigue confiando en los protocolos de actuación. Sin embargo llama la atención que en este punto no se aluda a las armas químicas. ${ }^{44}$

\subsection{A Strong Britain in an Age of Uncertaintv: The National Security Strategv}

Para el Reino Unido la principal amenaza de las armas de destrucción masiva podría venir vinculada al terrorismo y especialmente a $\mathrm{Al}$ Qaeda ${ }^{45}$. Sin embargo, no considera que éste sea

\footnotetext{
37 "Elle appelle de ses voeux la généralisation de ces efforts au niveau international. À cet effet, elle soutient activement l'entrée en vigueur du Traité d'interdiction complète des essais nucléaires et l'engagement de négociations multilaterales sur un traité d'interdiction de la production de matières fissiles à des fins d'armes nucléaires, ainsi que la mise en place d'un moratoire immédiat sur la production de ces matières”, en Ibid., p. 76.

38 "The American people face no greater or more urgent danger than a terrorist attack with a nuclear weapon", en "National Security Strategy", White House (2011), p. 23.

39 "Black markets trade in nuclear secrets and materials. Terrorists are determined to buy, build, or steal a nuclear weapon", en Ibid., p. 23.

40 "To strengthen the NPT, we will seek more resources and authority for international inspections. We will develop a new framework for civil nuclear cooperation", en Ibid., Idem.

41 "Pursue the Goal of a World Without Nuclear Weapons", en Ibid., Idem.

42 "We will pursue ratification of the Comprehensive Test Ban Treaty. And we will seek a new treaty that verifiably ends the production of fissile materials intended for use in nuclear weapons”, en Ibid., Idem.

43 "As countries move increasingly to tap peaceful nuclear energy to provide power generation while advancing climate goals, the world must develop an infrastructure in the countries that seek to use nuclear energy for their energy security needs and climate goals to ensure that nuclear energy is developed in a safer manner", en Ibid., Idem.

44 "The effective dissemination of a lethal biological agent within a population center would endanger the lives of hundreds of thousands of people and have unprecedented economic, societal, and political consequences", en Ibid., p. 24.
} 
el único problema que tiene que afrontar el Reino Unido ya que califica a la proliferación como un problema creciente $^{46}$ De hecho, apunta directamente a Irán como un Estado Proliferador que puede desestabilizar toda la región de Oriente Medio con especial incidencia en el proceso de $\mathrm{Paz}^{47}$.

En cuanto a la forma de solucionar este problema aboga por la cooperación con otros Estado, tanto desde el punto de vista bilateral como desde el punto de vista multilateral ${ }^{48}$

Por último el documento cuenta con una metodología muy bien elaborada que está detallada en su anexo final lo que aporta un valor añadido a las previsiones que se hacen sobre el futuro de la seguridad del Reino Unido.

\subsection{Strong and Secure. A Strategv for Australia's National Securitv ${ }^{49}$}

Australia está re-elaborando sus documentos de Seguridad Nacional. Uno de ellos es la Estrategia de Seguridad Nacional, un documento bastante bien elaborado donde las Armas de Destrucción Masiva ocupan un lugar privilegiado. Australia distingue entre:
a. National Security Objectives
b. Key National Security Risks
c. Pillar of Australia Security.

Dentro de los "Key National Security Risks" se establece como una categoría la lucha contra la proliferación nuclear ${ }^{50}$ lo cual nos da una idea de la importancia que concede Australia a este asunto. Debido precisamente a esta importancia alta, Australia considera que la proliferación fundamental es uno de los pilares básicos de la seguridad del Estado.

Se podría pensar que Australia no está rodeado de ningún Estado proliferador. Sin embargo, el documento que estamos analizando desvincula la amenaza del elemento territorial y establece que se trata de una amenaza global que no debe ser entendida bajo la lógica de la cercanía geográfica.

\footnotetext{
45 "Al Qaeda and other groups have stated an aspiration to develop unconventional (chemical, biological, radiological or nuclear - CBRN) capabilities" "A Strong Britain in an Age of Uncertainty: The National Security Strategy", Ministry of Defence, Londres (Octubre 2011), p. 28

46 "Proliferation is a growing danger", en Ibid., p. 3.

47 "If Iran acquires nuclear weapons technology, there is a strong possibility that other states in the region would follow", en Ibid., p.14.

48 "Yet they are indispensable to global solutions on issues such as climate change and nuclear proliferation", en Ibid., p.15.

${ }^{49}$ Un trabajo crítico sobre la Doctrina de Seguridad de Australia en: Dupond, Alan y Reckmeyer, Williams: “Australia's national security priorities: addressing strategic risk in a globalised world", Australian Journal of International Affairs, vol. 66, $\mathrm{n}^{\circ} 1$ (February 2012).

50 "Strong and Secure. A Strategy for Australia's National Security", Australian Government-Office of the Prime Minister, Canberra, (2013) p.2, en http://www.dpmc.gov.au/national_security/docs/national_security_strategy.pdf.
} 
Por último y siguiendo con el tradicional multilateralismo australiano, la Estrategia de Seguridad de Australia establece que el Consejo de Seguridad de las Naciones Unidas es el órgano encargado de tratar ésta y otras amenazas no tradicionales ${ }^{51}$.

\section{Conclusiones}

El primero de los problemas que tiene tanto el documento de 2011 como el de 2013 es que no define que entiende por Armas de Destrucción Masiva. Se hace necesaria o bien una definición o cuanto menos aceptar algunas de las más aceptadas a nivel internacional. De este modo, podemos entender a qué tipo de armas nos estamos refiriendo.

En general parece claro que los dos documentos que hemos analizados no conceden a la proliferación de armas de destrucción masiva la importancia que merece. Nuestros aliados son especialmente cuidadosos con esta cuestión. En el caso de los Estados Unidos incluso se desarrollan algunos documentos específicos, sobre los que hemos venido trabajando, como son el "Joint Doctrine for Combating Weapons of Mass Destruction" "2, "National Military Strategy to Combat Weapons of Mass Destruction", con el fin específico de combatir este problema $^{53}$. Sin embargo, llama la atención que el documento desarrollado por el Real Instituto Elcano establece como una de las prioridades de la seguridad la lucha contra la proliferación nuclear ${ }^{54}$.

En definitiva se puede decir que mientras que los Estados Unidos tienen una estrategia planificada para luchar contra la proliferación de armas de destrucción masiva, en España o bien no la hay o al menos no se hace pública. En el documento norteamericano se distingue entre "Means" "Ways" y "Ends" lo que permite un análisis más acertado de la realidad así como una estrategia probablemente más eficaz. Dentro de los medios se agrupan a todas las capacidades de los Estados Unidos así como la participación de sus aliados. Para los modos se destaca la división entre pilares ${ }^{55}$, objetivos militares estratégicos ${ }^{56}$ (MSO) y áreas de misión ${ }^{57}$. El objetivo final de estas estrategias no es otro que lograr la seguridad de los Estados Unidos, usando para ello tanto a sus fuerzas armadas como la de sus aliados. Si bien es cierto que los Estados cuentan con los aliados para frenar la proliferación, no es menos cierto que tanto en la

\footnotetext{
51 “support the Council's work to counter terrorism, the proliferation of weapons of mass destruction, and piracy”, en Ibid., p. 36.

52 "Joint Doctrine for Combating Weapons of Mass Destruction", Federation of American Scientist (FAS), Washington D.C, en http://www.fas.org/irp/doddir/dod/jp3_40.pdf.

53 "National Military Strategy to Combat Weapons of Mass Destruction", Department of Defense, Washington D.C. (2006), en http://www.defense.gov/pdf/nms-cwmd2006.pdf.

${ }^{54}$ Molina, Ignacio: "Hacia una renovación estratégica de la política exterior española", Real Instituto Elcano (2014), p. 52, en http://www.realinstitutoelcano.org/wps/portal/web/rielcano_es/publicacion?WCM_GLOBAL_CONTEXT=/elca no/elcano_es/publicaciones/informe-elcano-15-renovacion-politica-exterior-espana.

${ }^{55}$ En este punto se destaca la no-proliferación, la contra-proliferación así como la gestión de las consecuencias.

56 En este punto destacamos la prevención, la reducción/destrucción/reverso, la derrota/disuasión y la defensa/respuesta y recuperación.

${ }^{57}$ Se destacan 8 áreas de misión entre las que destaca la Seguridad y Cooperación y Actividades Asociadas, Prohibición de las Armas de destrucción masiva, las operaciones ofensivas, Operaciones de reducción de la cooperación, la eliminación de las Armas de Destrucción Masiva, Defensa Activa, Defensa Pasiva así como la gestión de las consecuencias.
} 
Doctrina de Seguridad Nacional de 2002 como en la de 2006 se citaba expresamente la protección de los aliados por parte de los Estados Unidos ${ }^{58}$.

Si nos fijamos en los documentos de otros Estados, en la mayor parte de ellos encontramos alusiones a la no-proliferación más detalladas y mejor estructuras. Otro ejemplo que no hemos señalado anteriormente es el caso de Finlandia quien no está en una zona geográfica con grandes amenazas - con la excepción rusa- relacionadas con las Armas de Destrucción Masiva pero que sin embargo es consciente del problema ${ }^{59}$. Si acudimos a un Estado con un problema cercano, como puede ser el caso de Japón, nos damos cuenta que aquí la importancia es mucho mayor ${ }^{60}$. En todo caso, la importancia que los Estados conceden a la proliferación de armas de destrucción masiva es mucho más que la que se le atribuye en el caso de España, donde además se relativiza la amenaza que suponen las armas químicas y las bacteriológicas.

\footnotetext{
58 "Goal V, to prevent our enemies from threatening us, our allies and our friends with WMD, repeats the 2002 principle that the duty to protect the American people obligates the government to anticipate and counter threats, using all elements of national power, before the threats can do grave damage", en Gay, Christine: "The Bush Doctrine Revisited: The 2006 National Security Strategy of the USA" Chinese Journal of International Law, vol. $5, \mathrm{n}^{\circ} 3$ (2006), p. 562.

59 "Finnish Security and Defence Policy", Prime Minister Office, Helsinki (2012), p.44.

60 "National Security Strategy", Japan Prime Minister, Tokyo (17 de diciembre del 2013), p.2.
} 
GRÁFICO 1: Marco de la lucha contra las Armas de Destrucción Masiva

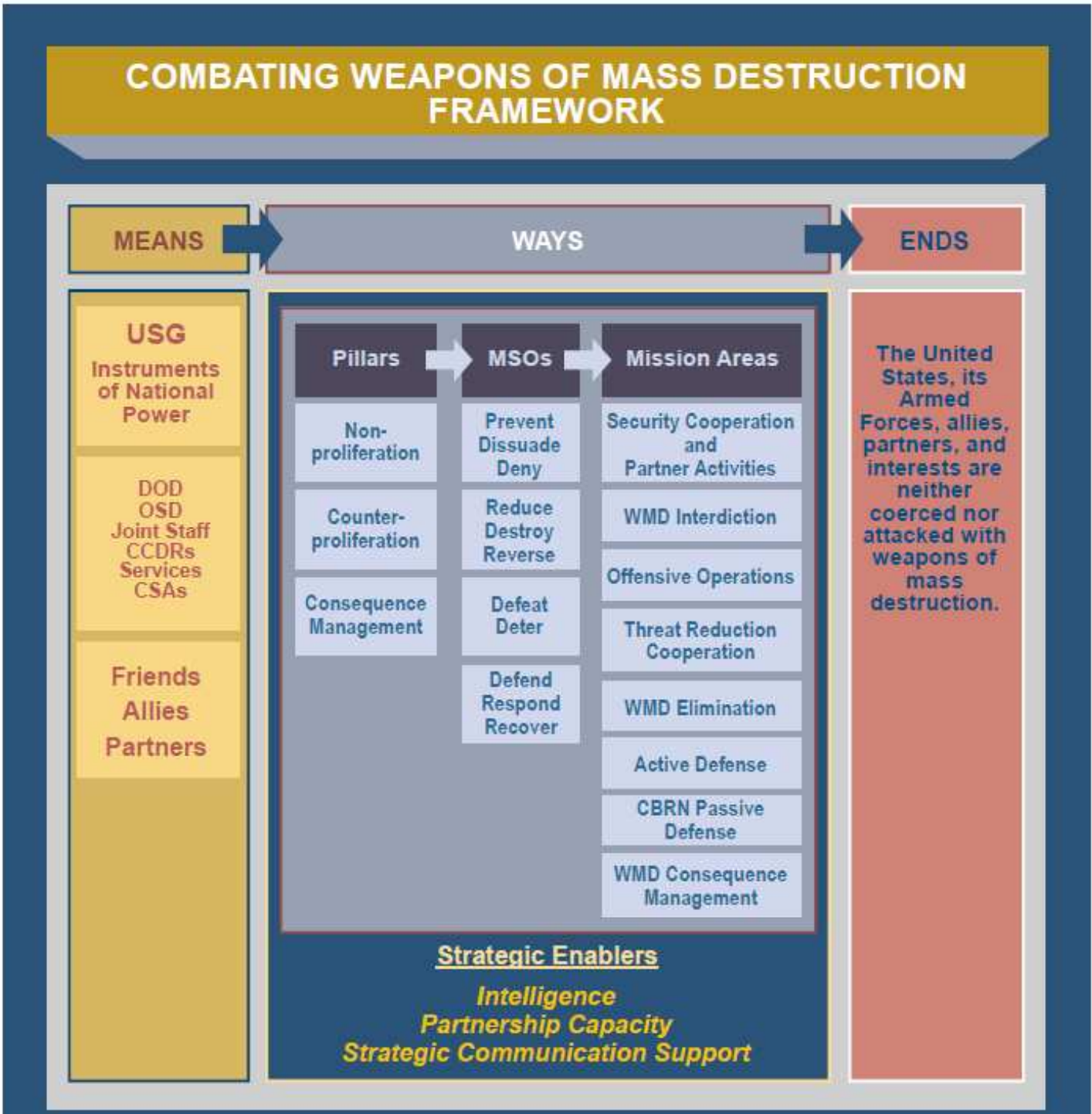

FUENTE: US Joint Chief of Staff

Precisamente en el ámbito internacional la coordinación de la posición española con las doctrinas de UE y OTAN es cuanto menos insuficiente. En cualquiera de los dos casos no se hace alusión ni de forma directa ni de forma indirecta a los documentos marco (Conceptos Estratégicos o Documento Solana) que guían el comportamiento de estas organizaciones en el ámbito de la seguridad. Tampoco se han señalado las iniciativas o herramientas llevadas a cabo en el seno de la OTAN y de la UE y que tienen por principal objetivo la lucha contra la proliferación. Por ello, parece que la acción de España en este campo está descoordinada tanto de sus aliados como de las organizaciones de seguridad a las que pertenece.

Otro de los elementos que debería haberse mencionado en los documentos que estamos analizando es la revisión del TNP llevada a cabo en 2010. Si bien es cierto que otros trabajos hablan de los puntos de avance y retroceso de este proceso, nuestros documentos hacen caso omiso al mismo. Incluso tampoco se ha menciona la posición conjunta de la UE en dicha conferencia. 
También se puede decir que la distinción entre riesgos y amenazas está poco detallada en el documento español. En el resto de los documentos que hemos analizado esta distinción queda más clara por lo que consideramos que podría ser mejorado.

Otro de los aspectos que se podrían señalar es la ausencia de una metodología clara en la elaboración del documento español. Todos los documentos con los que la hemos comparado están muy por encima del español desde el punto de vista metodológico.

Por ello, desde el punto de vista general se trata de un documento mejorable y desde el punto de vista de la proliferación creemos que no se le concede la importancia que merece. Probablemente se considera que España no tiene vecinos proliferadores pero esto es un claro error ya que como se destaca en otras doctrinas la base territorial no sirve como modelo de análisis en mundo cada vez más globalizado y, en el caso del Mediterráneo, del resultado de las negociaciones con Irán y del mantenimiento o no del TNP, dependerá el nacimiento de una importante cadena de Estados proliferantes nucleares que justamente pueden llegar a la frontera sur de España.

En lo que a la tendencia de la proliferación se refiere vivimos y vamos a vivir en un mundo donde cada vez más habrá un número más elevado de armas nucleares. Si bien es cierto que algunos Estados como Francia o el Reino Unido pueden estar pensando en la reducción del número de cabezas nucleares, otros como India o Pakistán están pensando precisamente en todo lo contrario. Los dos Estados de subcontinente tienen como objetivo alcanzar las 400 cabezas nucleares lo que les convertiría en la cuarta y quinta potencia nuclear solo por detrás del Reino Unido, Rusia y los Estados Unidos ${ }^{61}$. No obstante, después de las invasiones de Irak, Georgia o Ucrania parece que lo único que disuade a los Estados es la posesión de Armas de Destrucción Masiva. Por ello, el líder de la comunidad tártara de Crimea ha señalado que la única opción que tiene Ucrania para sobrevivir es la renuclearización como elemento de disuasión frente a Rusia. Además, siguiendo a algunos expertos en materia de desarme como Hans $\mathrm{Blix}^{62}$, este asunto parece no estar en la agenda de los grandes líderes por lo que el escenario al que nos enfrentamos no es muy diferente al que nos describía John Mearsheimer en su famoso artículo "Back to the future"

\footnotetext{
61 "According to Riedel, Pakistan is even on course to become the fourth-largest nuclear-weapons state, ahead of France, which is deemed to have 300 weapons", en Fitzpatrick, Mark (2014): Overcoming Pakistan's nuclear dangers, Londres, IISS, p. 21.

62 "A more important impediment, however, is that the nuclear-weapons states no longer seem to take their commitment to nuclear disarmament seriously”, en Blix, Hans (2008): Why Nuclear Disarmament Matters, Cambridge (Mass), The MIT Press, p. 11.
} 